\title{
Changes in Risk Factors and Exercise Capacity After Cardiac Rehabilitation and Its Effect on Hospital Readmission
}

\author{
Farid Najafi ${ }^{1, *} ;$ Mehdi Nalini $^{2, *} ;$ Mohammad Reza Nikbakht $^{3}$ \\ ${ }_{1}^{1}$ Research Center for Environmental Determinants of Health, Kermanshah University of Medical Sciences, Kermanshah, IR Iran \\ 2 Imam Ali Heart Center, Kermanshah University of Medical Sciences, Kermanshah, IR Iran \\ ${ }^{3}$ School of Pharmacy, Kermanshah University of Medical Sciences, Kermanshah, IR Iran \\ ${ }^{*}$ Corresponding Authors: Mehdi Nalini, Vice Chancellery of Research and Technology, Kermanshah University of Medical Sciences, Building No 2, Shahid Beheshti Blvd, Kermanshah, \\ IR Iran. Tel:+98-9183853465, Fax:+98-8318392834, E-mail: nalinimehdi@yahoo.co.uk; Farid Najafi, Vice Chancellery of Research and Technology, Kermanshah University of Medical \\ Sciences, Building No 2, Shahid Beheshti Blvd, Kermanshah, IR Iran. Tel:+98-9183853465, Fax:+98-8318392834, E-mail: fnajafi@kums.ac.ir
}

Received: May 13, 2012; Revised: January 28, 2013; Accepted: February 15, 2014

\begin{abstract}
Background: Despite the positive outcomes reported with cardiac rehabilitation(CR), its impacts have been reported to be different from a region or country to another, which may be due to the different contents of rehabilitation programs.

Objectives: To investigate the effect of CRon cardiovascular risk factors.

Patients and Methods: This is a retrospective cohort study on the data from Imam Ali Cardiac Rehabilitation Center in Kermanshah province, Iran from 2001 to 2008 . We used paired t-test to evaluate the effect of CR on cardiovascular risk factors. Logistic regression or t-test (unequal variance) were used to assess the factors influencing re-admission (due to cardiac problems). The relationship between different variables and death was studied using univariate cox proportional hazard. P values $<0.05$ were considered significant for all analyses.

Results: Out of 504 patients who completed rehabilitation, a total of 499 were analyzed. These 499 patients consisted of 383 men and 116 women. All anthropometric measurements, blood lipids (except HDL cholesterol), systolic and diastolic blood pressure, depression, anxiety and exercise capacity improved after rehabilitation $(\mathrm{P}<0.05$ for all cases). The improvement was observed in both sexes. A total of 39 patients were re-admitted to hospital after rehabilitation. Being female $(\mathrm{OR}=2.40 ; 95 \% \mathrm{CI}: 1.22-4.68)$ and history of diabetes $(\mathrm{OR}=2.04$; 95\%CI:1.04-4.02)increased the risk of re-admission significantly. Patients who were readmitted had higher anthropometric measurements at the beginning and the end of the program. Moreover, the initial exercise capacity of readmitted patients was lower than those who were not readmitted. After a maximal follow-up period of 6.3 years (median $=2.99$ years), only eight patients expired (survival rate: $97.5 \%$; $95 \%$ CI: 94.7-98.8). None of the variables in our study was significantly related to the survival rate.

Conclusions: The comprehensive CR program in Imam Ali Center efficiently reduces cardiovascular risk factors and improves exercise capacity.
\end{abstract}

Keywords:Rehabilitation; Exercise Tolerance; Patient Readmission; Risk Factors

\section{Background}

Cardiovascular diseases constitute the number one cause of death worldwide. Cerebral and cardiac ischemic diseases are the two most common causes of death in all countries (whether high- or low-income) and thus it has been estimated that they will continue to top the list of mortality causes until 2030 (1). In Iran and other developing countries which are experiencing an epidemiologic transition, with the disease pattern shifting from communicable to non-communicable and chronic diseases, the prevalence of cardiac disease is rising rapidly. According to the first study on burden of diseases in Iran in 2003, cardiovascular diseases constitute the third cause of disability adjusted life years (DALYs) after intentional and unintentional injuries and psychiatric and behav- ioral disorders. Out of 2267 DALYs per 100000 people, 780 (34.5\%) years are years lived with disability (YLD). This fact highlights the role of secondary prevention alongside primary prevention for cardiovascular diseases (2).

Cardiac rehabilitation (CR) includes all the activities aimed at helping patients with cardiovascular disease to achieve their optimal physical, mental and social conditions, so that they may pursue their normal roles in the society and maintain an active life. Thus, the objective of $\mathrm{CR}$ is to reduce recurrence of disease and its mortality, improve clinical improvement through controlled exercises, modify lifestyle and educate about health. Exercise, lifestyle changes such as quitting smoking, blood lipid control, blood pressure control, weight control and

Implication for health policy/practice/research/medical education:

Despite wide use of cardiac rehabilitation in cardiovascular centers and hospitasl, it is still under-used in developed and developing countries. There are many reasons for this under-utilization. However, one of the most important factors related to its underuse is low referral rate by physicians due to the fact that their primary focus is on other procedures rather than lifestyle changes. This study adds to the existing evidence regarding the positive effects of cardiac rehabilitation.

Copyright (C) 2014, Iranian Red Crescent Medical Journal; Published by Kowsar Corp. This is an open-access article distributed under the terms of the Creative Commons Attribution License, which permits unrestricted use, distribution, and reproduction in any medium, provided the original work is properly cited. 
treating diabetes, as well as psychological instructions are among interventions which efficiently influence patient's relative recovery. Studies indicate that as a result of these interventions, $\mathrm{CR}$ reduces recurrence and disability, and improves quality of life and reduces mortality considerably as well (3-6).

Despite the positive outcomes reported with CR, it has been different from one region or country to another, and even different hospitals of one country have observed different results. These differences may be due to the content of CR program. On the other hand, CR programs with identical content may differ in terms of frequency and duration, and these differences will undoubtedly account for the observed discrepancies in terms of effect on cholesterol, High-density lipoprotein (HDL), Low-density lipoprotein (LDL) and blood pressure (3-12).

\section{Objectives}

Since no study has been conducted so far to evaluate the rehabilitation programs conducted at this center with their impacts on different health factors, as well as the fact that the programs are conducted relatively differently and the majority of patients are post-operative cardiac patients, we designed and conducted the present study.

\section{Patients and Methods}

The Imam Ali CR Center is located in Kermanshah in western Iran. It has initiated its activity since 2001 and is currently the exclusive CR center in western Iran, covering patients even from neighboring provinces. This is a retrospective cohort study on the data from Imam Ali Cardiac Rehabilitation Center. The participants included all patients who were referred to this CR center from 2001 to 2008 and completed its programs.

The CR program consists of medical and pharmaceutical evaluation, supervised exercise, and education and consultation for cardiovascular risk factors modification. The educational sessions address nutrition, medical, exercise (mostly endurance including threadmil, walking and stationary bicycle) and psychological issues. The program is presented as a comprehensive course (three sessions per week for two months, with a total of 26 sessions) or a short course (one session per week for two months, with a total of 10 sessions).

At the beginning of each course, the patients are evaluated for serum lipids, body mass index, waist circumference, blood pressure, depression, anxiety, smoking, diabetes, and exercise capacity in metabolic equivalents (METs) through maximal symptom limit exercise test. For the calculation of METs, we used modified Bruce protocol. Their information was recorded in their medical files. Once the CR program was completed, these evaluations were repeated and the changes were recorded again. All laboratory tests for blood lipids were performed in the laboratory of Imam Ali Hospital. In this study, data were extracted from patients' medical files. Moreover, cases of readmission for cardiac problem and mortality (all-cause mortality) were identified through telephone calls made to patients or their families. All patients who had been readmitted were invited to refer to Imam Ali CR Center with their medical records. If the patients had moved from their previous address, we tried to locate them via friends or the telephone directory. Finally, those patients who could not be traced were searched for in the death registry of Kermanshah Province so that if expired, their date of death was recorded.

In the present study, we classified patients as hypertensive when they declared being hypertensive and received treatment for hypertension, or those with diastolic and systolic blood pressures (at the beginning of the program) of equal to or more than 90 and $140 \mathrm{mmHg}$, respectively. Regarding diabetes, patients were labeled diabetic if they declared to be diabetic and received insulin or oral medicine, or those with fasting blood sugar levels of equal to or more than $126 \mathrm{mg} / \mathrm{dL}$ at the beginning of the program.

We used Beck's scale for diagnosis of depression. The patients were categorized in four groups in terms of severity of depression: no depression (0-9), mild depression (10-15), moderate depression (16-23), and severe depression (24-63). Spielberg's scale was used for anxiety, categorizing patients as having no stress or mild stress (2032), moderate stress (33-52), or severe stress (53-80). While Spielberg's questionnaire can evaluate both the state and the trait of anxiety, in order to evaluate the effect of 2-3 months of the CR program we only used the "state" part.

In the present study, changes in anthropometric characteristics and cardiovascular risk factors, which constituted a continuous quantitative measurement, were assessed using paired t-test on STATA software. The relationship between variables and readmission (due to cardiac diseases) was evaluated using logistic regression for nominal or ranked variables. For the continuous quantitative variables, the mean values in two groups (with and without readmission) were compared using t-test (unequal variance). In order to evaluate mortality, first the survival rate curve was plotted for all patients. Considering the small number of expired patients during the study period, it was impossible to study the relationship between different variables and patient mortality using a multivariate model and therefore we used univariate cox proportional hazard method to determine the relationship between major variables and death. P values $<0.05$ were considered significant for all analyses.

The present study was approved by ethics committee of Kermanshah University of Medical Sciences in 2009. After complete follow-up of patients till next event (hospital admission and/or death) we deleted all identifiers such as name, family name and father's name.

\section{Results}

A total of 581 patients were referred to Imam Ali Center for CR from 2001 to 2008. Out of these patients, $504 \mathrm{com}$ - 
pleted the program; however, five patients were excluded from the study because of missing demographic data. Thus, a total of 499 patients were analyzed, consisting of 383 men and 116 women.

The men and women were not significantly different in terms of mean age; however, the number of non-smokers, the illiterate, and patients with hypertension and diabetes were significantly higher among women (Table 1). The majority of patients $(87.8 \%)$ had been referred to the rehabilitation center following coronary artery bypass grafting surgery (CABG) or percutaneous coronary intervention (PCI); these patients constituted $66.4 \%$ of women, which was significantly lower compared to men (Table 1 ).

\subsection{Improvement in Risk Factors and Exercise Ca- pacity}

A comparison of risk factors and exercise capacity before and after rehabilitation indicated that all anthropometric measurements, systolic and diastolic blood pressure, blood lipids (except HDL), depression, anxiety, and exercise capacity improved after rehabilitation program ( $\mathrm{P}<0.05$ for all cases) (Table 2 ). This improvement except for HDL was observed for both sexes (data is not present- ed in tables). In addition, the type of CR (8 sessions or 24 sessions) did not affect the magnitude of improvement considerably ( $\mathrm{P}>0.05$ for all cases).

\subsection{Readmission and Its Related Factors}

Out of 499 patients referred for rehabilitation, 40 patients were readmitted to hospital for cardiac reasons; one patient was readmitted prior to initiation of rehab program, and was therefore excluded from the analysis. In the present study, factors of hypertension, smoking, education level, age, depression, stress, and marital status were not related to readmission (Table 3). On the other hand, female sex (OR $=2.40$; 95\%CI: 1.22-4.68) and history of diabetes $(\mathrm{OR}=2.04 ; 95 \% \mathrm{CI}: 1.04-4.02)$ significantly increased the risk of readmission (Table 3). Furthermore, a comparison of mean anthropometric measurements, exercise capacity, and mean values of blood lipids in patients with or without readmission indicated that readmitted patients had larger anthropometric measurements at the beginning and the end of CR. Although the exercise capacity of readmitted patients was initially lower compared to those who were not readmitted, no statistically significance difference in exercise capacity was observed at the end of the rehabilitation program (Table 4).

Table 1. Comparison of Demographic and Risk Factors Among Men and Women Participating in the Cardiac Rehabilitation Program $^{\mathrm{a}, \mathrm{b}}$

\begin{tabular}{|c|c|c|c|c|}
\hline $\begin{array}{l}\text { Demographic and Risk } \\
\text { Factors }\end{array}$ & Male $(\mathbf{n}=383)$ & Female $(n=116)$ & Pvalue & Total $(n=499)$ \\
\hline Age & $53.9 \pm 9.0$ & $54.8 \pm 8.0$ & 0.32 & $54.1 \pm 8.8$ \\
\hline Smoking & & & $<0.001$ & \\
\hline Never & 26.1 & 89.4 & & 41.1 \\
\hline Cessation & 68.4 & 8.9 & & 54.3 \\
\hline Active & 5.5 & 1.8 & & 4.6 \\
\hline Married & 98.4 & 80.8 & $<0.001$ & 94.3 \\
\hline Education & & & $<0.001$ & \\
\hline Illiterate & 9.9 & 38.8 & & 16.6 \\
\hline Elementary & 34.7 & 40.5 & & 36.1 \\
\hline Diploma & 25.3 & 12.9 & & 22.4 \\
\hline University & 30.1 & 7.8 & & 24.9 \\
\hline Indication & & & $<0.001$ & \\
\hline CABG/PCI & 94.3 & 66.4 & & 87.8 \\
\hline CAD & 5.0 & 24.1 & & 9.4 \\
\hline Valve surgery & 0.7 & 5.2 & & 1.8 \\
\hline High risk for CAD & 0.0 & 4.3 & & 1.0 \\
\hline Diabetes & 20.4 & 35.3 & 0.001 & 23.9 \\
\hline Hypertension & 35.0 & 54.2 & $<0.001$ & 48.5 \\
\hline Cardiac Rehabilitation Type & & & 0.004 & \\
\hline 10 sessions & 13.8 & 4.3 & & 11.6 \\
\hline 26 sessions & 86.2 & 95.7 & & 88.4 \\
\hline
\end{tabular}


Najafi F et al.

\begin{tabular}{|c|c|c|c|}
\hline Variables & Before Rehabilitation & After Rehabilitation & Pvalue \\
\hline Weight, kg & $73.5 \pm 10.3$ & $71.8 \pm 10.0$ & $<0.001$ \\
\hline Body Mass Index, $\mathrm{kg} / \mathrm{m}^{2}$ & $26.5 \pm 3.8$ & $25.9 \pm 3.6$ & $<0.001$ \\
\hline Waist, cm & $95.4 \pm 9.3$ & $92.1 \pm 9.0$ & $<0.001$ \\
\hline Systolic Blood Pressure, $\mathrm{mmHg}$ & $128.6 \pm 17.7$ & $117.3 \pm 11.7$ & $<0.001$ \\
\hline Diastolic Blood Pressure, $\mathrm{mmHg}$ & $82.2 \pm 10.6$ & $76.2 \pm 7.9$ & $<0.001$ \\
\hline Depression (Beck score) $^{c}$ & $14.0 \pm 8.7$ & $9.3 \pm 6.5$ & $<0.001$ \\
\hline Anxiety (Spielberg score) ${ }^{\mathrm{C}}$ & $40.2 \pm 11.0$ & $36.7 \pm 8.9$ & $<0.001$ \\
\hline Exercise Capacity (METs) & $9.4 \pm 2.6$ & $11.4 \pm 2.7$ & $<0.001$ \\
\hline Total Cholesterol, mg/dL & $211.3 \pm 47.0$ & $190.4 \pm 42.9$ & $<0.001$ \\
\hline LDL Cholesterol, mg/dL & $132.2 \pm 36.0$ & $116.6 \pm 37.7$ & $<0.001$ \\
\hline HDL Cholesterol, mg/dL & $41.2 \pm 12.6$ & $41.8 \pm 13.5$ & 0.31 \\
\hline Triglyceride, mg/dL & $192.6 \pm 81.9$ & $161.4 \pm 73.2$ & $<0.001$ \\
\hline
\end{tabular}

Table 3. Relationship Between Different Variables and Readmission After Completion of $\mathrm{CR}^{\mathrm{a}}$

\begin{tabular}{lll}
\hline Variables & OR & CI 95\% \\
\hline Age $\geq \mathbf{5 5}$ & 0.65 & $0.33-1.26$ \\
Gender (Female) & 2.4 & $1.22-4.68$ \\
\hline Education & & \\
\hline illiterate & 1.00 & - \\
\hline elementary & 0.59 & $0.24-1.45$ \\
\hline Diploma & 0.47 & $0.16-1.36$ \\
\hline University & 0.96 & $0.39-2.37$ \\
Marriage & 0.47 & $0.17-1.30$ \\
\hline Diabetes & 2.04 & $1.04-4.02$ \\
\hline Hypertension & 0.89 & $0.43-1.80$ \\
\hline Cardiac Rehabilitation Type & & \\
\hline 10 sessions & 1.00 & - \\
\hline 26 sessions & 2.19 & $0.51-9.47$ \\
\hline Smoker (at start of program) & & \\
\hline Never & 1.00 & - \\
\hline Cessation & 0.62 & $0.31-1.21$ \\
\hline active & 0.88 & $0.19-4.05$ \\
\hline Indication & & \\
\hline CABG/PCI & 1.00 & - \\
\hline CAD & 2.70 & $1.16-6.26$ \\
\hline Valve surgery & - & \\
\hline High risk for CAD & 3.28 & $0.36-30.27$ \\
\hline Depression status & & \\
\hline Without depression & 1.00 & - \\
\hline Mild depression & 0.90 & $0.38-2.10$ \\
\hline Moderate depression & 1.30 & $0.52-3.20$ \\
\hline Severe depression & 1.63 & $0.58-4.60$ \\
\hline Anxiety status & & \\
\hline Without or mild anxiety & 1.00 & - \\
\hline Moderate anxiety & 0.80 & $0.32-2.00$ \\
\hline Severe anxiety & $0.29-3.84$ \\
\hline abbreviations: CABG, coronary artery bypass graft; CAD, coronary \\
artery diseases; CI, confidence interval; CR, cardiac rehabilitation; OR, \\
odds ratio; PCI, percutaneous coronary intervention. & \\
\hline
\end{tabular}

Table 4. Comparison of Anthropometric Measurements and Exercise Capacity in Patients With and Without Readmission a,b

\begin{tabular}{|c|c|c|c|}
\hline Variables & $\begin{array}{l}\text { With Read- } \\
\text { mission }\end{array}$ & $\begin{array}{l}\text { Without Re- } \\
\text { admission }\end{array}$ & Pvalue \\
\hline Weight before CR, kg & $76.6 \pm 10.3$ & $73.2 \pm 10.3$ & 0.04 \\
\hline Weight after CR, kg & $74.9 \pm 10.0$ & $71.6 \pm 9.9$ & 0.05 \\
\hline BMI before CR, $\mathrm{kg} / \mathrm{m}^{2}$ & $28.3 \pm 4.0$ & $26.3 \pm 3.7$ & 0.004 \\
\hline BMI after $\mathrm{CR}, \mathrm{kg} / \mathrm{m}^{2}$ & $27.6 \pm 4.0$ & $25.8 \pm 3.6$ & 0.007 \\
\hline Waist before $\mathrm{CR}, \mathrm{cm}$ & $100.0 \pm 9.2$ & $95.0 \pm 9.3$ & 0.002 \\
\hline Waist after CR, cm & $96.0 \pm 8.6$ & $91.7 \pm 9.0$ & 0.004 \\
\hline $\begin{array}{l}\text { Exercise capacity } \\
\text { before CR(METs) }\end{array}$ & $8.6 \pm 2.8$ & $9.6 \pm 2.6$ & 0.04 \\
\hline $\begin{array}{l}\text { Exercise capacity } \\
\text { after CR(METs) }\end{array}$ & $10.9 \pm 2.9$ & $11.4 \pm 2.7$ & 0.33 \\
\hline
\end{tabular}

\subsection{Survival Rate in Referred Patients}

After a maximal follow-up period of 6.3 years (median $=2.99$ years, mean $=3.27 \pm 1.74$ years), only eight patients expired (survival rate $=97.5$ years; $95 \% \mathrm{CI}=94.7-98.8$ ), consisting of seven men and one woman. Among these, six patients were referred to the CR center following CABG/ PCA. None of the variables in our study were significantly related to survival rate in the univariate analysis.

\section{Discussion}

The present study indicated that CR in combination with other interventions can efficiently improve the modifiable cardiovascular risk factors, except HDL. Furthermore, it was indicated that history of diabetes and female sex are two risk factors for readmission in patients referred for $\mathrm{CR}$.

Previous studies have indicated different results for the effect of comprehensive $\mathrm{CR}$ on mean blood lipids values. 
Najafi Fet al.

While some clinical trials have suggested that rehabilitation reduces total cholesterol effectively (7-10), others have failed to indicate a statistically significant effect (11, 12). This is also true for LDL and triglyceride levels (10-12). Most previous studies have indicated that CR does not affect HDL levels significantly $(7,12,13)$. Similarly, the present study found that CR reduces all blood lipids except HDL, and this is a mechanism for reducing the risk of cardiovascular disease and death in these patients. Nevertheless, it must be noted that despite all these reports, which sometimes differ regarding the effect of CR on blood lipids, meta-analysis by Taylor et al. (2004) indicated that rehabilitation improves total cholesterol, LDL and triglyceride levels while leaving HDL nearly unchanged (5). In fact exercise and possible changes in nutritional pattern of patients during rehabilitation are among major factors which reduce blood lipids.

Several points must be made about the anthropometric changes. In the present study, all anthropometric measurements (weight, waist circumference and body mass index) improved. Although relatively small (about $2 \%$ ), the changes were statistically significant. As a matter of fact, many CR programs integrate weight control and thus it is expected that patients lose $10 \%$ weight (1-2 pounds per week) over a six-month period (14). However, it is noteworthy that many reports indicate that this weight loss is not feasible in short term $(15,16)$ and even in some cases, particularly for obese and overweight patients, the patients may initially gain some weight (17, 18). Previous studies have reported a maximum of $1 \%$ to $\%$ weight loss (19-21) which is consistent with our findings.

Our study showed that CR has an impressive blood pressure-lowering effect: $11.3 \mathrm{mmHg}$ for systolic and 6.0 $\mathrm{mmHg}$ for diastolic blood pressure. This study confirms the findings of previous studies that $\mathrm{CR}$ reduces both systolic and diastolic blood pressure $(9,22)$. A meta-analysis including 54 clinical trials showed that after aerobic exercise program, systolic and diastolic blood pressure reduced significantly (3.9 and $2.6 \mathrm{~mm} \mathrm{Hg}$ respectively). However, our findings are in contrast to another metaanalysis (5) that showed exercise based cardiac rehabilitation doesn't improve diastolic blood pressure. The reasons for this discrepancy can be attributed to methodological differences among different studies. Findings of one study indicate that while exercise alone is effective in reducing blood pressure, the addition of a behavioral weight loss program significantly augments the efficacy of aerobic training (23). Another study showed that diastolic blood pressure changes positively correlate with serum cholesterol and insulin resistance (24) which can be enhanced during comprehensive CR programs. The effect of CR on blood pressure needs to be further investigated in future prospective studies including proper control groups.

An important finding of the present study is the improved exercise capacity by $25 \%$. After cardiac events, the METs level has been a shown to be a significant predictor of final prognosis of patients (25). According to the American Heart Association report, CR for 3 to 6 months generally increases exercise capacity by $11 \%$ to $36 \%$ (26). Improvement of exercise capacity after CR has been shown in numerous studies (13, 27-30).

The present study shows an improvement in depression and anxiety following CR program which is in line with findings of previous studies $(21,31,32)$. Enhancement of anxiety and depression have a major impact on medical outcomes in patients with cardiovascular disease by reducing morbidity and mortality as well as improving the quality of life (33). It is important to note that we evaluated the state of anxiety in a way that we evaluates how the patients feel at the moment of evaluation. The responses can vary with the feelings of patients. In fact there are different reasons for observed improvement in depression and anxiety of patients. One important reason can be related to the content of program. We run the CR program within a group of patients and therefore it provides a positive atmosphere for better interaction with each other. In addition, most of people referred to this center are after CABG. Such patients have passed the recovery period and CR program can improve their feeling of well-being. We also studied the factors related to readmission of patients after completion of CR program. Numerous studies have dealt with the effect of CR on readmission (34-36). It seems that factors influencing readmission in patients undergoing CR cannot be very different from factors influencing readmission after a cardiovascular event. Most studies have incriminated diabetes as a factor affecting the survival rate and subsequent hospital admissions of different types of cardiac patients $(37,38)$. Similarly, some studies have reported poorer prognosis and higher risk of readmission for female patients $(38,39)$. This is quite consistent with our findings which indicate a higher rate of readmission for female and diabetic patients.

The survival rate was $97.5 \%$ in the present study. As a matter of fact, although previous studies have yielded controversial results regarding the effect of CR on survival rate in patients with cardiac events, most recent studies suggest a positive role for CR in terms of reducing mortality $(4,5,40,41)$. Since our study does not entail a control group, we were unable to compare the survival rate in our patients with those who did not take CR, yet comparing our findings with other studies suggests that survival in our patients (mostly patients who underwent $\mathrm{CABG} / \mathrm{PCI}$ ) is relatively higher compared to patients who do not participate in cardiac rehabilitation $(42,43)$.

\subsection{Strengths and Weaknesses}

The present study is the largest study conducted in western Iran, where the only CR center covers Kermanshah and its neighboring provinces, thus its findings are of immense value. Nonetheless, due to the short duration of follow-up and the low incidence of outcomes, it cannot identify the influential factors, which require further 
studies with longer durations of follow-up. On the other hand, we were unable to control the treatments that patients received during rehabilitation (which were often changed by physicians) and include them in our analysis. We are not able to determine the exact contribution of changes in medication in the observed improvements. In addition, because of no control group one may discuss that the observed improvement in risk factors might be the effect of factors other rather than CR. Prospective studies with inclusion of control group may circumvent this problem to a large extent. In addition, due to the small number of cases for some variables (e.g. active smokers among women), some of the statistical tests did not have sufficient power.

In spite of the low popularity of CR programs among cardiologists in countries like Iran, such programs can efficiently reduce cardiovascular risk factors and improve exercise capacity in patients with heart disease. The outcomes of these programs in Imam Ali CR center in Kermanshah are comparable to CR programs in other centers. Such findings are in line with other studies to show that cardiologists' fears are not rational. Though not certainly, we can believe that CR plays an important role in reducing the risk factors of CVD. History of diabetes as well as being female both increased the risk of readmission for cardiac reasons.

\section{Acknowledgements}

We would like to thank all staffs and doctors working in cardiac rehabilitation center of Imam Ali hospital.

\section{Authors' Contribution}

Farid Najafi: design of study, analysis, contribution to the first draft and approval of final draft. Mehdi Nalini: contribution in design of study, preparation of first draft and approval of final draft. Mohammad Reza Nikbakht: added experience to the whole work, contribution in final draft.

\section{Financial Disclosure}

The authors declare that there are no conflicts of interest.

\section{Funding/Support}

This study was funded by Kermanshah University of Medical Sciences.

\section{References}

1. World Health Organization. Cardiovascular disease. Fact sheet $N$ 317. WHO; 2011. Available from: http://www.who.int/mediacentre/ factsheets/fs317/en/.

2. Ministry of Health and Medical Education. National Burden of Disease Study in Iran, 1993: Ministry of Health and Medical Education, Deputy Ministry for Health; 1996.

3. Braverman DL. Cardiac rehabilitation: a contemporary review. Am JPhys Med Rehabil. 2011;90(7):599-611.
4. Heran BS, Chen JM, Ebrahim S, Moxham T, Oldridge N, Rees K, et al. Exercise-based cardiac rehabilitation for coronary heart disease. Cochrane Database Syst Rev. 2011;(7):CD001800.

5. Taylor RS, Brown A, Ebrahim S, Jolliffe J, Noorani H, Rees K, et al. Exercise-based rehabilitation for patients with coronary heart disease: systematic review and meta-analysis of randomized controlled trials. Am J Med. 2004;116(10):682-92.

6. Wenger NK. Current status of cardiac rehabilitation. J Am Coll Cardiol. 2008;51(17):1619-31.

7. Engblom E, Ronnemaa T, Hamalainen H, Kallio V, Vanttinen E, Knuts LR. Coronary heart disease risk factors before and after bypass surgery: results of a controlled trial on multifactorial rehabilitation. Eur Heart J.1992;13(2):232-7.

8. Carlsson R, Lindberg G, Westin L, Israelsson B. Influence of coronary nursing management follow up on lifestyle after acute myocardial infarction. Heart. 1997;77(3):256-9.

9. Silberman A, Banthia R, Estay IS, Kemp C, Studley J, Hareras D, et al. The effectiveness and efficacy of an intensive cardiac rehabilitation program in 24 sites. Am J Health Promot. 2010;24(4):260-6.

10. Lakusic N, Mahovic D, Ramqaj T, Cerovec D, Grbavac Z, Babic T. The effect of 3-weeks stationary cardiac rehabilitation on plasma lipids level in 444 patients with coronary heart disease. Coll Antropol. 2004;28(2):623-9.

11. Belardinelli R, Paolini I, Cianci G, Piva R, Georgiou D, Purcaro A Exercise training intervention after coronary angioplasty: the ETICA trial. J Am Coll Cardiol. 2001;37(7):1891-900.

12. Toobert DJ, Glasgow RE, Radcliffe JL. Physiologic and related behavioral outcomes from the Women's Lifestyle Heart Trial. Ann Behav Med. 2000;22(1):1-9.

13. Yu CM, Li LS, Ho HH, Lau CP. Long-term changes in exercise capacity, quality of life, body anthropometry, and lipid profiles after a cardiac rehabilitation program in obese patients with coronary heart disease. Am J Cardiol. 2003;91(3):321-5.

14. Ades PA, Balady GJ, Berra K. Transforming exercise-based cardiac rehabilitation programs into secondary prevention centers: a national imperative. J Cardiopulm Rehabil. 2001;21(5):263-72.

15. Savage PD, Lee M, Harvey-Berino J, Brochu M, Ades PA. Weight reduction in the cardiac rehabilitation setting. J Cardiopulm Rehabil. 2002;22(3):154-60.

16. Harvey-Berino J. Weight loss in the clinical setting: applications for cardiac rehabilitation. Coron Artery Dis. 1998;9(12):795-8.

17. McConnell TR, Laubach CA, 3rd, Szmedra L. Age and Gender Related Trends in Body Composition, Lipids, and Exercise Capacity During Cardiac Rehabilitation. Am J Geriatr Cardiol.1997;6(4):37-45.

18. Pierson LM, Herbert WG, Norton HJ, Kiebzak GM, Griffith P, Fedor JM, et al. Effects of combined aerobic and resistance training versus aerobic training alone in cardiac rehabilitation.J Cardiopulm Rehabil. 2001;21(2):101-10.

19. Lavie CJ, Milani RV. Effects of cardiac rehabilitation, exercise training, and weight reduction on exercise capacity, coronary risk factors, behavioral characteristics, and quality of life in obese coronary patients. Am J Cardiol. 1997;79(4):397-401.

20. Bader DS, Maguire TE, Spahn CM, O'Malley CJ, Balady GJ. Clinical profile and outcomes of obese patients in cardiac rehabilitation stratified according to National Heart, Lung, and Blood Institute criteria. J Cardiopulm Rehabil. 2001;21(4):210-7.

21. Duarte Freitas P, Haida A, Bousquet M, Richard L, Mauriege P, Guiraud T. Short-term impact of a 4-week intensive cardiac rehabilitation program on quality of life and anxiety-depression. Ann Phys Rehabil Med. 2011;54(3):132-43.

22. Whelton SP, Chin A, Xin X, He J. Effect of aerobic exercise on blood pressure: a meta-analysis of randomized, controlled trials. Ann Intern Med. 2002;136(7):493-503.

23. Blumenthal JA, Sherwood A, Gullette EC, Babyak M, Waugh R, Georgiades A, et al. Exercise and weight loss reduce blood pressure in men and women with mild hypertension: effects on cardiovascular, metabolic, and hemodynamic functioning. Arch Intern Med. 2000;160(13):1947-58.

24. Brett SE, Ritter JM, Chowienczyk PJ. Diastolic blood pressure changes during exercise positively correlate with serum cholesterol and insulin resistance. Circulation. 2000;101(6):611-5

25. Arena R, Myers J, Williams MA, Gulati M, Kligfield P, Balady GJ 
et al. Assessment of functional capacity in clinical and research settings: a scientific statement from the American Heart Association Committee on Exercise, Rehabilitation, and Prevention of the Council on Clinical Cardiology and the Council on Cardiovascular Nursing. Circulation. 2007;116(3):329-43.

26. Leon AS, Franklin BA, Costa F, Balady GJ, Berra KA, Stewart KJ, et al. Cardiac rehabilitation and secondary prevention of coronary heart disease: an American Heart Association scientific statement from the Council on Clinical Cardiology (Subcommittee on Exercise, Cardiac Rehabilitation, and Prevention) and the Council on Nutrition, Physical Activity, and Metabolism (Subcommittee on Physical Activity), in collaboration with the American association of Cardiovascular and Pulmonary Rehabilitation. Circulation. 2005;111(3):369-76.

27. Hindman L, Falko JM, LaLonde M, Snow R, Caulin-Glaser T. Clinical profile and outcomes of diabetic and nondiabetic patients in cardiac rehabilitation. Am Heart J. 2005;150(5):1046-51.

28. Balady GJ, Jette D, Scheer J, Downing J. Changes in exercise capacity following cardiac rehabilitation in patients stratified according to age and gender. Results of the Massachusetts Association of Cardiovascular and Pulmonary Rehabilitation Multicenter Database. J Cardiopulm Rehabil.1996;16(1):38-46.

29. Manzoni GM, Villa V, Compare A, Castelnuovo G, Nibbio F, Titon AM, et al. Short-term effects of a multi-disciplinary cardiac rehabilitation programme on psychological well-being, exercise capacity and weight in a sample of obese in-patients with coronary heart disease: a practice-level study. Psychol Health Med. 2011;16(2):178-89.

30. Svacinova H, Novakova M, Placheta Z, Kohzuki M, Nagasaka M, Minami N, et al. Benefit of combined cardiac rehabilitation on exercise capacity and cardiovascular parameters in patients with type 2 diabetes. Tohoku J Exp Med. 2008;215(1):103-11.

31. Artham SM, Lavie CJ, Milani RV. Cardiac rehabilitation programs markedly improve high-risk profiles in coronary patients with high psychological distress. South Med J. 2008;101(3):262-7.

32. Yohannes AM, Doherty P, Bundy C, Yalfani A. The long-term benefits of cardiac rehabilitation on depression, anxiety, physical activity and quality of life. J Clin Nurs. 2010;19(19-20):2806-13.

33. Doerfler LA, Paraskos JA. Anxiety, posttraumatic stress disorder, and depression in patients with coronary heart disease: a practi- cal review for cardiac rehabilitation professionals. J Cardiopulm Rehabil. 2004;24(6):414-21.

34. Ades PA, Huang D, Weaver SO. Cardiac rehabilitation participation predicts lower rehospitalization costs. Am Heart J.1992;123(4 Pt1):916-21.

35. Carroll DL, Rankin SH, Cooper BA. The effects of a collaborative peer advisor/advanced practice nurse intervention: cardiac rehabilitation participation and rehospitalization in older adults after a cardiac event. J Cardiovasc Nurs. 2007;22(4):313-9.

36. Canyon S, Meshgin N. Cardiac rehabilitation - reducing hospital readmissions through community based programs. Aust Fam Physician. 2008;37(7):575-7.

37. Calafiore AM, Di Mauro M, Di Giammarco G, Contini M, Vitolla $\mathrm{G}$, Iaco AL, et al. Effect of diabetes on early and late survival after isolated first coronary bypass surgery in multivessel disease. $J$ Thorac Cardiovasc Surg. 2003;125(1):144-54.

38. Reina A, Colmenero M, Aguayo de Hoyos E, Aros F, Marti H, Claramonte R, et al. Gender differences in management and outcome of patients with acute myocardial infarction. Int J Cardiol. 2007;116(3):389-95.

39. Anand SS, Xie CC, Mehta S, Franzosi MG, Joyner C, Chrolavicius S, et al. Differences in the management and prognosis of women and men who suffer from acute coronary syndromes. J Am Coll Cardiol. 2005;46(10):1845-51.

40. Alter DA, Oh PI, Chong A. Relationship between cardiac rehabilitation and survival after acute cardiac hospitalization within a universal health care system. Eur J Cardiovasc Prev Rehabil. 2009;16(1):102-13.

41. Goel K, Lennon RJ, Tilbury RT, Squires RW, Thomas RJ. Impact of cardiac rehabilitation on mortality and cardiovascular events after percutaneous coronary intervention in the community. Circulation. 2011;123(21):2344-52.

42. Hannan EL, Racz MJ, McCallister BD, Ryan TJ, Arani DT, Isom OW, et al. A comparison of three-year survival after coronary artery bypass graft surgery and percutaneous transluminal coronary angioplasty. JAm Coll Cardiol. 1999;33(1):63-72.

43. Gao G, Wu Y, Grunkemeier GL, Furnary AP, Starr A. Long-term survival of patients after coronary artery bypass graft surgery: comparison of the pre-stent and post-stent eras. Ann Thorac Surg. 2006;82(3):806-10. 